\title{
Study of congenital fetal malformations among antenatal women in a tertiary care centre
}

\author{
Vibha Subhash More ${ }^{1}$, Annal Abhay Vaidya ${ }^{1 *}$, S. V. Parelkar ${ }^{2}$, Madhva Prasad $^{1}$
}

\begin{abstract}
${ }^{1}$ Department of Obstetrics and Gynaecology, ${ }^{2}$ Department of Paediatric Surgery, Seth Gordhandas Sunderdas Medical College and King Edward VII Memorial Hospital, Mumbai, Maharashtra, India
\end{abstract}

Received: 23 January 2018

Accepted: 28 February 2018

\author{
*Correspondence: \\ Dr. Annal Abhay Vaidya, \\ E-mail: annalvaidya911@gmail.com
}

Copyright: (c) the author(s), publisher and licensee Medip Academy. This is an open-access article distributed under the terms of the Creative Commons Attribution Non-Commercial License, which permits unrestricted non-commercial use, distribution, and reproduction in any medium, provided the original work is properly cited.

\begin{abstract}
Background: Congenital malformations are morphological or functional anomalies that occur in the prenatal period as a result of genetic mutation, chromosomal abnormalities and adverse intrauterine environment. Early recognition is important. If congenital malformations are detected beyond the period of legal limit for medical termination of pregnancy, it carries immense burden on the pregnant woman.

Methods: A descriptive observational study was conducted as a retrospective analysis of patient-records, over a span of 9 consecutive months in the Department of Obstetrics and Paediatric Surgery, at a tertiary care centre, Mumbai. The project was carried out to study demographic profiles, risk factors and system-wise distribution of pregnant women carrying malformed fetus.

Results: The average annual incidence rate was 29 per 1000 deliveries. Of 151 patients, renal malformations were the commonest accounted for $40 \%$ of all congenital malformations. This was followed by central nervous system $32 \%$, cardiovascular 14\%, gastrointestinal $8 \%$ and musculoskeletal $3 \%$. Congenital malformations were more common in multigravida than primigravida. $92 \%$ of congenital malformations were detected beyond 20 weeks of gestation. Previous abortions, hypothyroidism and raised sugars were associated high risk factors.

Conclusions: The diagnosis of congenital malformation using ultrasonography occurs at a later gestational age than 20 weeks. For these patients a combined approach of the obstetrician/neonatologist/paediatric surgeon allows better counselling of parents and to permit preparation of the team to optimize neonatal outcomes.
\end{abstract}

Keywords: Congenital malformations, Early recognition, Multidisciplinary approach

\section{INTRODCTION}

Congenital malformations are morphological abnormalities arising due to structural defects or abnormal formation of the tissues or organs. Worldwide, approximately 303,000 new-borns die within 4 weeks of life due to presence of a congenital malformation. These constitute one of the top 5 causes of neonatal deaths. ${ }^{1}$ The Indian scenario is quite similar. ${ }^{2}$ The proportional contribution of congenital malformations to infant mortality is also rising. Indian people have many risk factors for congenital malformations, including high fertility, unplanned pregnancy, consanguineous marriage, poor antenatal care, poor maternal nutritional status etc. It is important to diagnose congenital malformation during early antenatal period to allow medical termination of pregnancy if indicated.

It also allows adequate counselling of parents, to prepare the neonatal team including surgeons to optimize the outcome. The present study was carried out to study demographic profiles, risk factors and system-wise 
distribution of these congenital malformations among the patients who were antenatally diagnosed.

\section{METHODS}

This is a descriptive observational study, conducted as a retrospective analysis of patient-records over 9 months, in the Department of Obstetrics and Gynaecology in collaboration with Paediatric Surgery at a tertiary care centre in Mumbai.

A special outpatient clinic, "the birth defect clinic" is specifically designed to cater to the needs of currently pregnant women who are carrying a fetus with a diagnosed congenital malformation on radiological examination. All patients who come to the antenatal outpatient department with a diagnosed malformation are advised to attend the birth defect clinic. Here, the parents are counselled by a team of doctors which includes obstetrician, paediatric surgeon and neonatologist.

The objective of this clinic is to explain in detail regarding the nature of the congenital malformation, the prognosis and the possible interventions that may be required in the immediate postnatal period. This article describes the findings of all the patients who attended the clinic in the specified duration.

Records of all the patients, who were antenatally diagnosed with fetus having congenital malformations on ultrasonography within this period, were noted down. Cases were analysed for information regarding presence and nature of the congenital malformation, maternal age, parity, ultrasonography finding, risk factors in mother, consanguinity, bad obstetric history, medical and other obstetrical problems. The major malformations were divided into central nervous system, gastrointestinal, urinary, musculoskeletal, cardiovascular and miscellaneous disorder. Descriptive statistics were used for the study. Data was analysed for percentage distribution.

\section{RESULTS}

During the study period, 151 mothers with antenatal ultrasonography suggestive of fetal congenital malformation attended the birth defect OPD.

Table 1: Age and parity wise distribution.

\begin{tabular}{|lll|}
\hline Age (in years) & Number $\mathbf{N}=\mathbf{1 5 1}$ & Percentage \\
\hline$<20$ & 14 & 9.27 \\
\hline $21-25$ & 69 & 45.7 \\
\hline $26-30$ & 44 & 29.1 \\
\hline $31-35$ & 20 & 13.2 \\
\hline$>35$ & 6 & 3.97 \\
\hline Parity & $\mathbf{N}=\mathbf{1 5 1}$ & Percentage \\
\hline Primigravida & 59 & 39.07 \\
\hline Multigravida & 92 & 60.93 \\
\hline
\end{tabular}

During the study period, there were 5111 deliveries out of which 151 patients had antenatal ultrasonography suggestive of congenital malformations. The occurrence of congenital malformations was $2.9 \%$.

Out of 151 mothers, majority (86.75\%) 131 patients were between 21 to 35 years, $14(9.27 \%)$ were less or equal to 20 years and $6(3.97 \%)$ were more than 35 years old.

In the present study, congenital malformations were more in multigravida $92(60.93 \%)$ than Primigravida 59 $(39.07 \%)$

$63(41.72 \%)$ of the congenital malformations identified were severe and had poor prognosis; and 88 (58.27\%) had a relatively better prognosis. The gestational age at which the patient presented to the birth defect clinic is presented in Table 2 .

Table 2: Gestational age distribution.

\begin{tabular}{|ll|l|}
\hline Gestational age & Number & Percentage \\
\hline$<20$ & 12 & 7.9 \\
\hline $20-28$ weeks & 85 & 56.29 \\
\hline 28-34 weeks & 44 & 29.13 \\
\hline$>34$ weeks & 10 & 6.62 \\
\hline
\end{tabular}

Table 3: Detailed distribution of malformations.

\begin{tabular}{|lll|}
\hline Malformation & No. & $\%$ \\
\hline CNS & $\mathbf{4 8}$ & 31.78 \\
\hline Neural tube defects & 11 & 7.2 \\
\hline Ventriculomegaly & 7 & 4.6 \\
\hline Dandy-walker syndrome/Arnold- & 12 & 7.9 \\
Chiari malformation & & 5.9 \\
\hline Mega cisterna magna & 9 & 3.31 \\
\hline Multiple anomalies & 5 & 2.6 \\
\hline Others & 4 & 13.9 \\
\hline CVS & $\mathbf{2 1}$ & 3.31 \\
\hline Hypoplastic heart & 5 & 3.31 \\
\hline Complex congenital heart disease & 5 & 2.6 \\
\hline Tetralogy of Fallot & 4 & 1.32 \\
\hline Septal defects & 2 & 1.32 \\
\hline Dextrocardia with situs inverses & 2 & 1.98 \\
\hline Others & 3 & 40.3 \\
\hline Renal & $\mathbf{6 1}$ & 29.8 \\
\hline Pyelectesis & 45 & 5.9 \\
\hline Dysplastic kidneys & 9 & 1.98 \\
\hline Agenesis of unilateral kidneys & 3 & 1.32 \\
\hline Crossed fused pelvic kidneys & 2 & 1.32 \\
\hline Others & 2 & $\mathbf{8 . 6}$ \\
\hline Gastrointestinal & $\mathbf{1 3}$ & 1.98 \\
\hline Congenital diaphragmatic hernia & 3 & 3.9 \\
\hline Abdominal wall defects & 6 & 1.98 \\
\hline Duodenal atresia & 3 & 0.6 \\
\hline Oesophageal atresia & 1 & $\mathbf{2 . 6}$ \\
\hline Skeletal disorders & $\mathbf{4}$ & $\mathbf{2 . 6}$ \\
\hline Multiple systems involved & $\mathbf{4}$ & \\
\hline & & \\
\hline
\end{tabular}


System wise distributions of congenital malformations are summarized in Figure 1. Of 151 patients, renal malformations were the commonest $(40 \%)$. This was followed by central nervous system (32\%), cardiovascular (14\%), gastrointestinal (8\%) and musculoskeletal $(3 \%)$. The detailed distribution of the various malformations in each system is presented in Table 3.

Maternal risk factors observed have been shown in table 4. The most common factors were presence of previous abortion, hypothyroidism and abnormal sugars. 5 women $(3.31 \%)$ had history of congenital malformation in a prior pregnancy also. $13(8.6 \%)$ had polyhydramnios and 7 (4.6\%) had oligohydramnios.

\section{Table 4: Distribution according to risk factor.}

\begin{tabular}{|lll|}
\hline Risk factors & Number & Percentage \\
\hline Previous abortion & 14 & 9.2 \\
\hline Abnormal sugars & 12 & 7.9 \\
\hline Hypothyroidism & 11 & 7.2 \\
\hline Previous stillbirth & 8 & 5.2 \\
\hline Consanguineous marriage & 4 & 2.6 \\
\hline Twins & 5 & 3.31 \\
\hline Epilepsy on treatment & 5 & 3.31 \\
\hline Previous malformed fetus & 5 & 3.31 \\
\hline
\end{tabular}

\section{DISCUSSION}

Congenital malformations occur in almost 1 in every 44 pregnancies in India. ${ }^{3}$ This incidence is almost similar to the rate of occurrence of still births also, highlighting the importance of this condition. A large gap exists in the early detection of fetal malformations in India. ${ }^{4}$

In the present study, the occurrence of fetal congenital malformations was 295 per 10000 births. While this rate is somewhat similar to the rates reported by Bhide et al (238 per 10000) and Babu (380 per 10000), a much higher rate is observed when the population is observed at a lesser gestational age. ${ }^{5}$ Kahyap et al found a rate of 764 per 10000 , which is probably attributed to the fact that they chose only population who presented to their institute for an early ultrasonography.

In the present study, many patients were actually diagnosed of fetal congenital malformation elsewhere, but were referred for specialist management, mainly due to non-availability of expert obstetrician/neonatologist/paediatric surgeons who are well versed with management of specific conditions. Due to variation in referral patterns, the true frequency of occurrence of congenital malformations need further reconfirmation, especially in the light of non-availability of a national birth defect surveillance. ${ }^{3}$

The majority of the congenital malformations were observed in the age group of 21 to 30 years. While it has been shown that the incidence of malformations increases with age, recent studies implicate not only the maternal age, but also paternal age. Hence paternal age could be considered as a factor in future similar studies. ${ }^{6,7}$ More multigravida women presented with congenital malformations compared to primigravida women. Parity has been observed to be an independent risk factor for at least some specific types of congenital malformations. ${ }^{8}$

Only around $8 \%$ of the study population had presented to the birth defect clinic before 20 weeks of gestation. In the corresponding time period, 27 patients underwent medical termination of pregnancy for congenital malformations. In view of existing laws in India, i.e. the MTP Act, patients who presented after 20 weeks of gestation, cannot undergo termination of pregnancy even if the malformation carries substantial risk to the newborn. As we note in our study, $92 \%$ presented after 20 weeks of gestation. Even if we exclude those with nonsevere malformations, still a sizeable number $(42.4 \%)$ in our study were carrying a pregnancy with severe malformation in the fetus.

The corresponding proportion in the study by Kashyap et al was $66 \%$ (209 detected after 20 weeks among 312 total malformations). Similarly, in the study by Babu et al, in a 1000-consecutive pregnancy cohort, $24 \%$ of malformations were diagnosed as late as in the third trimester. Present study and these two studies serve to emphasize the alarming scenario which exists regarding the late diagnosis of fetal structural malformations.

In the present study, renal malformations were most common. However, the majority of these were accounted for by the relatively mild condition i.e. renal pyelectesis, majority of which resolve spontaneously and requires only close observation.

Excluding this condition, central nervous system anomalies were the most common accounting for $31.78 \%$. This is in congruence with other studies, such as that by Babu et al (45.94\% in a 1000 birth cohort) and Agarwal et al (24.3\%). ${ }^{9}$

The proportion of women with fetal congenital malformations who had previous abortions was $9.27 \%$. Almeida et al have shown that presence of previous abortions is a risk factor for congenital malformations.

The number of patients with recurrent malformations in this study was 5 and were all dissimilar compared to the previous nature of congenital malformation. In the population-based register study by Glinianaia et al, it has been estimated that the risk of recurrence of a similar malformation is around 2.5 times compared to a previous normal pregnancy and around 1.4 times more for a dissimilar malformation. ${ }^{10}$

Twenty patients had oligohydramnios/polyhydramnios associated with the congenital malformation. Almeida et 
al have also reported the association of liquor abnormalities with malformations. ${ }^{8}$

\section{CONCLUSION}

Worldwide, there has been a consistent drop in the infant mortality rate. While there has been a significant drop in the mortality rate due to infectious diseases and nutritional deficiencies, a proportionate reduction in the mortality due to congenital malformation is not noticed. All efforts should be made to this end. The first step would be for diagnosis of malformations for which appropriate intervention can be done at the earliest. Present study shows that majority of malformations are still being diagnosed at a late period of gestation. Urgent measures need to be taken to address this issue.

Funding: No funding sources

Conflict of interest: None declared

Ethical approval: The study was approved by the Institutional Ethics Committee

\section{REFERENCES}

1. WHO. Congenital anomalies. WHO Fact sheet. Available at: http://www.who.int/mediacentre/factsheets/fs370/en/

2. Gupta N, Kabra M, Kapoor S. Establishing national neonatal perinatal database and birth defects registry network - need of the hour! Indian Pediatr. 2014.51(9):693-6.

3. Bhide P, Gund P, Kar A. prevalence of congenital anomalies in an indian maternal cohort: healthcare, prevention, and surveillance implications. PLoS One. 2016;11(11):e0166408.

4. Kashyap N, Pradhan M, Singh N, Yadav S. Early detection of fetal malformation, a long distance yet to cover! present status and potential of first trimester ultrasonography in detection of fetal congenital malformation in a developing country: experience at a tertiary care centre in India. J Pregnancy. 2015;2015:623059.

5. Babu RS, Pasula S. Frequency of foetal anomalies in a tertiary care centre. J Clin Diagn Res. 2013;7(7):1276-9.

6. Savaskar SV, Mundada SK, Pathan AS, Gajbhiye SF. Study of various antenatal factors associated with congenital anomalies born at tertiary health centre. Int J Recent Trends Sci Techn. 2014;12(1):82-5.

7. Contia SL, Eisenberg ML. Paternal aging and increased risk of congenital disease, psychiatric disorders, and cancer. Asian J Androl. 2016;18(3):420-4.

8. Almeida L, Araujo Júnior E, Crott G, Okido M, Berezowski A, Duarte G, et al. Epidemiological risk factors and perinatal outcomes of congenital anomalies. Rev Bras Gynecol Obstet. 2016;38(7):348-55.

9. Agarwal A, Rattan KN, Dhiman A, Rattan A. Spectrum of congenital anomalies among surgical patients at a tertiary care centre over 4 years. Int $\mathbf{J}$ Pediatr. 2017;2017:4174573.

10. Glinianaia SV, Tennant PWG, Rankin J. Risk estimates of recurrent congenital anomalies in the UK: a population-based register study. BMC Med. 2017;15(1):20.

11. Rosano A, Botto LD, Botting B. Infant mortality and congenital anomalies from 1950 to 1994: an international perspective. J Epidemiol Comm Health. 2000;54:660-6.

Cite this article as: More VS, Vaidya AA, Parelkar SV, Prasad M. Study of congenital fetal malformations among antenatal women in a tertiary care centre. Int J Reprod Contracept Obstet Gynecol 2018;7:1505-8. 